\title{
Influência da Curva de Aprendizado no Sucesso e na Ocorrência de Complicações Associadas aos Procedimentos pela Via Radial
}

\author{
Gilberto Lahorgue Nunes ${ }^{1}$, Alessandra Teixeira de Oliveira' ${ }^{1}$, \\ Leonardo Alves ${ }^{2}$, Trajano Alfonso ${ }^{3}$
}

\section{RESUMO}

Fundamentos: A via de acesso transradial é uma técnica empregada mais recentemente na realização de procedimentos percutâneos coronários (diagnósticos e terapêuticos), sendo que apresenta várias vantagens em relação ao acesso femoral. Apesar disto, o acesso radial tem sido empregado de rotina apenas em uma minoria dos laboratórios de hemodinâmica de nosso país. Objetivo: Determinar a influência da curva de aprendizado sobre o índice de sucesso e a incidência de complicações associadas à realização tanto de procedimentos diagnósticos quanto terapêuticos pela via radial. Método: Foram analisados 3.500 pacientes consecutivos submetidos a procedimentos pela via radial, no período de abril de 2000 a junho de 2003. Foram excluídos desta análise pacientes com teste de Allen anormal e em programa de hemodiálise. O efeito da curva de aprendizado sobre os índices de sucesso e ocorrência de complicações foi avaliado comparando-se os resultados obtidos nos primeiros 500 pacientes (Grupo I) com os observados nos restantes 3000 pacientes (Grupo II). Resultados: Não houve diferença entre os grupos no que diz respeito ao sexo, à idade e ao tipo de procedimento realizado (diagnóstico ou terapêutico). A taxa de sucesso foi significativamente superior $(98,1 \%$ contra $95,2 \%, p<0,01)$ e a ocorrência de complicações associadas ao sítio de acesso vascular significativamente menor no Grupo II (2,4\% contra $6,1 \%$, $\mathrm{p}<0,01)$. Adicionalmente, foi observado aumento progressivo dos índices de sucesso do procedimento com o incremento da experiência. Desta forma, o sucesso na realização do procedimento pela via radial nos primeiros 50, 100, 500 e 1.000 casos foi de, respectivamente, 88\%, 94\%, 97 e 98\%. Conclusão: A técnica radial está associada a uma curva de aprendizado longa, que excede os primeiros quinhentos pacientes, sendo que a incidência de sucesso aumenta e a ocorrência de complicações diminui, de maneira significativa, com o aumento do número de casos.

DESCRITORES: Artéria radial. Coronariopatia. Angiografia. Angioplastia. Coronary angiography, métodos. Competência clínica. Aprendizagem. Cateterismo periférico, métodos.

\footnotetext{
Serviço de Cardiologia Intervencionista - Hospital São Francisco, Porto Alegre, RS.

2 Serviço de Hemodinâmica do Hospital de Cardiologia, Santa Casa de Rio Grande, Porto Alegre, RS.

Correspondência: Gilberto L. Nunes. Rua Prof. Annes Dias, 285 Centro - Porto Alegre - RS - CEP 90020-090

E-mail: gilberto.nunes@terra.com.br

Recebido em: 1/4/2007 • Aceito em: 7/5/2007
}

SUMMARY

Influence of the Learning Curve on the Success and Occurrence of Complications Associated with Transradial Procedures

Background: The transradial approach is a more recent technique employed to perform diagnostic and therapeutic coronary procedures, which offers many advantages when compared to the femoral approach. Nevertheless, the radial access is routinely utilized in only a few catheterization laboratories in our country. Objective: To determine the influence of the learning curve on the success rate and incidence of complications associated with performing diagnostic and therapeutic procedures using the radial approach. Methods: We analyzed 3,500 consecutive patients submitted to transradial procedures from April, 2000 through June, 2003. Patients with an abnormal Allen test, absent radial pulse, and those undergoing hemodialysis were excluded from this analysis. The effect of the learning curve on the success rate and on the occurrence of complications was evaluated by comparing the results obtained in the first 500 patients (Group I) to the results achieved in the remaining 3,000 patients (Group II). Results: There were no differences identified between the groups in regard to gender, age and type of procedure performed (diagnostic catheterization or percutaneous intervention). The success rate was significantly higher $(98.1 \%$ versus $95.2 \%, \mathrm{p}<0.01)$ and the incidence of complications related to the vascular access site significantly smaller $(2.4 \%$ versus $6.1 \%, \mathrm{p}<0.01)$ in Group II. Additionally, a progressive increase in the success rate was observed with the greater experience using the technique. Therefore, the success rates in performing the procedure through the radial approach in the first 50,100, 500 and 1,000 cases were, respectively, $88 \%, 94 \%, 97 \%$ and $98 \%$. Conclusions: The transradial approach is associated with a long learning curve that exceeds the first 500 cases. The incidence of success increases and the occurrence of complications decreases significantly with the increase of the caseload.

DESCRIPTORS: Radial artery. Coronary disease. Angiography. Angioplasty. Coronary angiography, methods. Clinical competence. Learning. Catheterization, peripheral, methods. 
Nunes GL, et al. Influência da Curva de Aprendizado no Sucesso e na Ocorrência de Complicações Associadas aos Procedimentos pela Via Radial. Rev Bras Cardiol Invas 2007; 15(2): 115-118.

D esde a descrição inicial da técnica de cateterismo radial por Campeau', em 1989, o acesso transradial tem sido utilizado com freqüência crescente em laboratórios de hemodinâmica de todo mundo e, também, no nosso meio. Apesar disto, a maioria dos operadores apresenta experiência limitada com esta técnica, visto que ela tende a ser utilizada por muitos operadores apenas em casos selecionados e não como técnica primordial na realização de procedimentos coronários.

O emprego da técnica transradial está associado a algumas vantagens, como a possibilidade de deambulação imediata do paciente e a redução da ocorrência de complicações vasculares relacionadas ao sítio da punção. Por outro lado, a artéria radial possui calibre menor e está mais sujeita à ocorrência de espasmo do que a artéria femoral, que é o acesso vascular mais comumente utilizado. Desta forma, tanto a punção quanto a manipulação dos cateteres são mais difíceis em comparação à técnica de Judkins. Devido a estas dificuldades técnicas, o acesso transradial está associado a uma curva de aprendizado significativa, sendo que o número de casos necessários para se obter proficiência nesta técnica ainda permanece indefinido ${ }^{2}$.

O objetivo do presente estudo foi determinar a influência da curva de aprendizado sobre o índice de sucesso e a ocorrência de complicações associadas à realização de procedimentos diagnósticos ou terapêuticos pela via radial.

\section{MÉTODO}

Foram analisados 3.500 pacientes consecutivos submetidos a cinecoronariografia diagnóstica ou intervenção coronária percutânea utilizando o acesso transradial, em nossa instituição, no período de abril de 2000 a junho de 2003. Estes procedimentos foram realizados por uma equipe constituída de três cardiologistas intervencionistas.

Foram consideradas contra-indicações absolutas para o emprego da via radial a ausência do pulso radial, a presença de teste de Allen anormal (não perfusão da mão dentro de 10 segundos após a liberação da compressão sobre a artéria ulnar) ou de fístula arteriovenosa para realização de hemodiálise.

A punção era realizada com o membro superior direito estendido ao lado do corpo do paciente e com a palma da mão virada para cima. Aplicava-se anestesia local com 2 a $3 \mathrm{ml}$ de xilocaína e a artéria radial era puncionada utilizando-se abocath 22. Após a introdução da bainha arterial, eram injetados intra-arterialmente 200 mcg de nitroglicerina e 2 a 3 mg de verapamil. Além disto, 5.000 UI de heparina eram administradas em uma veia periférica. A sedação (diazepam ou fentanil EV) era realizada a critério do operador, sendo geralmente empregada apenas em pacientes apresentando quadro de ansiedade intensa.

Os procedimentos diagnósticos foram realizados utilizando-se, de modo geral, cateteres 5F (multipropósito, preferentemente, ou cateteres pré-moldados) e as intervenções coronárias empregando-se cateteres-guia 6 ou 7F. Após a realização do procedimento, a bainha arterial era imediatamente retirada (sem reversão da heparina e independente do emprego concomitante de antagonistas da glicoproteína IIb/IIla), sendo aplicado um curativo compressivo local (composto de gazes e bandagem elástica tipo Tensoplast $\left.{ }^{\circledR}\right)$. Na maioria dos casos, o paciente deixava a sala de cateterismo caminhando ou em cadeira de rodas, sendo permitida a livre movimentação durante o período de observação. O curativo compressivo local era retirado, geralmente, 3 a 4 horas após o final do procedimento.

Foram avaliados os índices de sucesso (definido como realização completa do procedimento pela via radial) e a incidência de complicações associadas ao sítio de acesso vascular. Visando a avaliar o impacto da curva de aprendizado sobre estes parâmetros, os pacientes foram divididos em dois grupos: Grupo I (composto dos primeiros 500 pacientes da nossa experiência) e Grupo II (os restantes 3.000 pacientes).

\section{RESULTADOS}

A idade média dos pacientes era semelhante nos dois grupos (situando-se em torno dos 59 anos), sendo que a maioria deles era do sexo masculino (Tabela 1). Aproximadamente $20 \%$ dos pacientes foram submetidos a intervenção coronária percutânea e, os restantes, a cinecoronariografia diagnóstica.

O sucesso do procedimento foi significativamente menor e a incidência de complicações significativamente maior nos primeiros 500 casos da nossa casuística

TABELA 1

Características demográficas e do procedimento

\begin{tabular}{lccc}
\hline & Grupo I (n=500) & Grupo II (n=3.500) & p \\
\cline { 1 - 2 } Idade (média) & $58 \pm 11$ anos & $59 \pm 11$ anos & NS \\
Sexo feminino & $212(42,4 \%)$ & $1393(39,8 \%)$ & NS \\
Cateterismo diagnóstico & $404(80,8 \%)$ & $2879(79,3 \%)$ & NS \\
Intervenção percutânea & $96(19,2 \%)$ & $621(20,7 \%)$ & NS \\
\hline
\end{tabular}


Nunes GL, et al. Influência da Curva de Aprendizado no Sucesso e na Ocorrência de Complicações Associadas aos Procedimentos pela Via Radial. Rev Bras Cardiol Invas 2007; 15(2): 115-118.

(Tabela 2). Com o aumento da experiência com a técnica radial, a ocorrência de insucesso por falha na punção ou espasmo importante apresentou um significativo declínio. Adicionalmente, observou-se que a ocorrência de hematoma local significativo foi reduzida em mais de 4 vezes com o aumento do número de casos (Tabela 2).

As Figuras 1 e 2 mostram o comportamento do índice de sucesso e da ocorrência de qualquer tipo de complicação durante ou após o procedimento (incluindo espasmo significativo, hematoma de qualquer porte, infecção local, surgimento de fístula arteriovenosa ou pseudo-aneurisma), de acordo com o aumento do número de casos. Nota-se um "plateau" da curva de aprendizado a partir do $500^{\circ}$ caso.

\section{DISCUSSÃo}

O acesso transradial representa uma técnica extremamente elegante de realização de procedimentos coronários percutâneos, quer diagnósticos ou terapêuticos. No nosso serviço, a introdução desta técnica (no início do ano 2000) permitiu uma mudança significativa da rotina de realização dos procedimentos, sendo que o aumento da experiência permitiu que o acesso femoral fosse utilizado com freqüência gradativamente decrescente, tornando-se a punção radial a nossa via de acesso preferencial. Desta forma, atualmente, o acesso radial é empregado em 75 a $80 \%$ dos casos realizados na nossa instituição. A abordagem radial apresenta inúmeras vantagens em relação à via femoral, como o maior

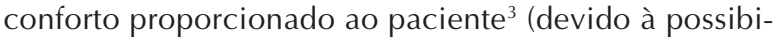
lidade de deambulação imediata, sem necessidade de repouso em decúbito dorsal), menor risco de complicações hemorrágicas locais ${ }^{4}$ e menor tempo de observação nos procedimentos diagnósticos ambulatoriais ${ }^{3}$. Além disto, a retirada mais precoce da bainha (associada ao menor risco de ocorrência de complicações hemorrágicas locais ${ }^{5}$ ) permite que, na maioria dos casos,

TABELA 2

Resultados

\begin{tabular}{lccc}
\hline & $\begin{array}{c}\text { Grupo I } \\
(\mathbf{n = 5 0 0})\end{array}$ & $\begin{array}{c}\text { Grupo II } \\
(\mathbf{n = 3 . 5 0 0 )}\end{array}$ & $\mathbf{p}$ \\
\hline Sucesso & $95,2 \%$ & $98,1 \%$ & $<0,001$ \\
Insucesso & $4,8 \%$ & $1,9 \%$ & $<0,001$ \\
Causas do insucesso & & & \\
$\quad \begin{array}{c}\text { Tortuosidade/oclusão } \\
\quad 1,6 \%\end{array}$ & $1 \%$ & $\mathrm{NS}$ \\
$\quad$ Espasmo importante & $0,6 \%$ & $0,1 \%$ & 0,02 \\
$\quad$ Falha na punção & $2,2 \%$ & $0,6 \%$ & $<0,001$ \\
Complicações* & $6,1 \%$ & $2,4 \%$ & $<0,001$ \\
$\quad$ Hematoma local & $2,2 \%$ & $0,5 \%$ & $<0,001$
\end{tabular}

*Espasmo importante, hematoma local de qualquer porte, infecção, fístula arteriovenosa, pseudo-aneurisma. se prescinda do encaminhamento do paciente à UTI ou unidade semi-intensiva após a realização de procedimentos terapêuticos, o que reduz de maneira significativa os custos hospitalares ${ }^{6}$. Finalmente, esta técnica possibilita, inclusive, a realização de intervenções coronárias percutâneas de forma ambulatorial ${ }^{7}$.

Entretanto, apesar destas vantagens, a punção radial é empregada como via de acesso primordial apenas em uma minoria dos laboratórios de hemodinâmica ao redor do mundo e, também, no nosso país. A maior razão para isto é a existência de uma curva de aprendizado associada a esta técnica, mesmo quando realizada por operadores com larga experiência no acesso femoral, sendo que a duração desta curva de aprendizado ainda é motivo de debate $^{2,8-10}$.

A curva de aprendizado associada à técnica radial envolve vários fatores, incluindo alguns aspectos técnicos peculiares a esta via de acesso. O primeiro deles é a punção da artéria radial. Ao contrário do acesso femoral, a anestesia local é realizada com pequena quantidade de anestésico local, aplicado não diretamente sobre o pulso radial, mas ao redor dele, visando "congelar" a artéria radial e facilitar a posterior punção. Também a seleção do material de punção é importante. Temos preferido utilizar cateter tipo Jelco ou Abocath 22", pois estes dispositivos possuem fino calibre e

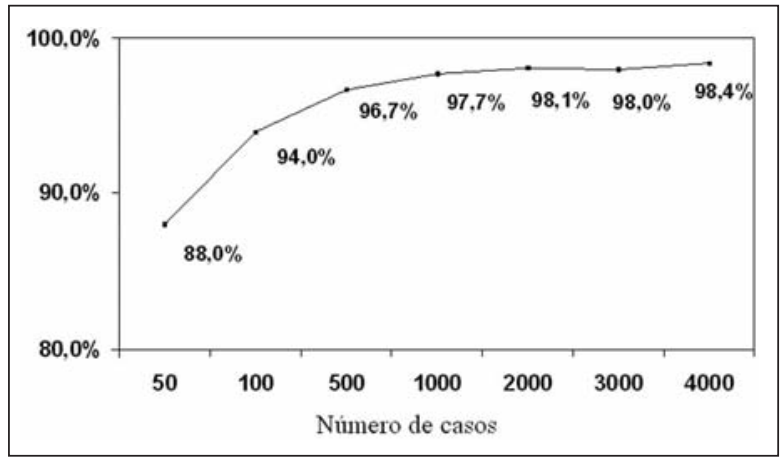

Figura 1 - Evolução do sucesso.

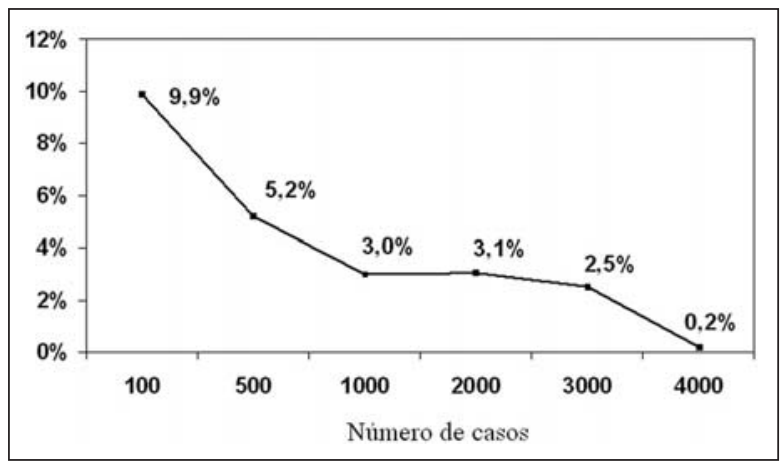

Figura 2 - Evolução das complicações. *Espasmo importante, hematoma de qualquer porte, infecção, fístula $A-V$, pseudo-aneurisma. 
paredes moles e, conseqüentemente, estão associados a uma menor incidência de surgimento de hematoma local no caso de uma primeira tentativa de punção não bem sucedida. Outro aspecto importante diz respeito à seleção dos cateteres. Ao contrário do observado com a via femoral, existe grande vantagem em realizar-se a cinecoronariografia utilizando-se apenas um único cateter. Isto é especialmente importante nos pacientes com artérias radiais pequenas ou que possuam grande tortuosidade nas artérias radial, braquial ou subclávia, visto que a troca freqüente de cateteres pode favorecer o surgimento de espasmo importante, dificultar a progressão do cateteres e, conseqüentemente, causar significativo desconforto ao paciente. Além disto, a utilização de apenas um cateter tem impacto positivo tanto sobre a duração do procedimento quanto sobre os custos associados. Na nossa instituição, aproximadamente $70 \%$ dos procedimentos diagnósticos são realizados empregando-se um único cateter multipropósito tipo MPA 5 F. No caso de impossibilidade de realização do exame com este cateter, cateteres pré-moldados (tipo Judkins de direita e esquerda e, eventualmente, tipo Amplatz) podem ser utilizados, tomando-se o cuidado de sempre selecionar um cateter de coronária esquerda com curva meio ponto menor do que o que seria utilizado se o procedimento fosse realizado por via femoral. Outros fatores importantes para o sucesso são a manipulação delicada dos cateteres, o emprego liberal de guias de troca mais rígidas tipo Amplatz 0.035" (especialmente quando há tortuosidade ou espasmo) e a realização de sedação, especialmente no início da experiência e em pacientes tensos e com tônus adrenérgico aumentado.

\section{CONCLUSÃO}

O presente estudo demonstra que a técnica transradial é segura e pode ser utilizada como via de acesso preferencial para realização de procedimentos coronários percutâneos, tanto diagnósticos com terapêuticos.
Entretanto, esta técnica está associada a uma curva de aprendizado significativa e que, como a nossa experiência demonstra, chega a abranger os primeiros 500 casos.

\section{REFERÊNCIAS BIBLIOGRÁFICAS}

1. Campeau L. Percutaneous radial artery approach for coronary angiography. Cathet Cardiovasc Diagn. 1989;16(1):3-7.

2. Louvard Y, Lefevre T, Morice MC. Radial approach: what about the learning curve? Cathet Cardiovasc Diagn. 1997;42 (4):467-8.

3. Cooper CJ, El-Shiekh RA, Cohen DJ, Blaesing L, Burket MW, Basu A, et al. Effect of transradial access on quality of life and cost of cardiac catheterization: a randomized comparison. Am Heart J. 1999;138(3 Pt 1):430-6.

4. Kiemeneij F, Laarman GJ, Odekerken D, Slagboom T, van der Wieken R. A randomized comparison of percutaneous transluminal coronary angioplasty by the radial, brachial and femoral approaches: the Access study. J Am Coll Cardiol. 1997;29(6):1269-75.

5. Choussat R, Black A, Bossi I, Fajadet J, Marco J. Vascular complications and clinical outcome after coronary angioplasty with platelet IIb/IIla receptor blockade. Comparison of transradial vs transfemoral arterial access. Eur Heart J. 2000;21(8): 662-7.

6. Mann JT $3^{\text {rd }}$, Cubeddu MG, Schneider JE, Arrowood M. Right radial access for PTCA: a prospective study demonstrates reduced complications and hospital charges. J Invasive Cardiol. 1996;8(suppl D):40D-4D.

7. Slagboom T, Kiemeneij F, Laarman GJ, van der Wieken R. Outpatient coronary angioplasty: feasible and safe. Catheter Cardiovasc Interv. 2005;64(4):421-7.

8. Goldberg SL, Renslo R, Sinow R, French WJ. Learning curve in the use of the radial artery as vascular access in the performance of percutaneous transluminal coronary angioplasty. Cathet Cardiovasc Diagn. 1998;44(2):147-52.

9. Louvard Y, Pezzano M, Sheers L, Koukoui F, Marien C, Benaim $R$, et al. Coronary angiography by a radial approach: feasibility, learning curve. One operator's experience. Arch Mal Coeur Vaiss. 1998;91(2):209-15.

10. Cheng TO. Influence of learning curve on the success of transradial coronary angioplasty. Cathet Cardiovasc Diagn. $1998 ; 45(2): 215-6$. 